\title{
Aplicación de la metodología aprendizaje-servicio en el Grado de Nutrición Humana y Dietética de la Universidad de Navarra
}

\author{
Susana Santiago \\ Roncesvalles Garayoa \\ Itziar Zazpe \\ Universidad de Navarra, España
}

\section{Resumen}

El aprendizaje-servicio es una metodología de innovación docente que permite aprender mediante la aproximación a la realidad. En las asignaturas de Educación Nutricional, Restauración Colectiva y Nutrición Comunitaria surge en el marco de la Innovación para la docencia impulsada dentro del Horizonte 2020 y se basa en experiencias previas en el Proyecto "Por una Comida sana, tú decides" y TANTAKA. Un total de 303 alumnos de la Facultad de Farmacia y Nutrición de la Universidad de Navarra han realizado asesoramiento dietético y actividades formativas en instituciones navarras (España).

\section{Palabras clave}

Sello aprendizaje-servicio, asesoramiento dietético, actividades formativas, instituciones navarras. 


\title{
Application of the service-learning methodology in the Degree in Human Nutrition and Dietetics at the University of Navarra
}

\begin{abstract}
Service-learning is an innovative teaching methodology that fosters learning through an approach to reality. In the subjects of Nutritional Education, Collective Restoration and Community Nutrition, a service-learning project was conceived within the framework of teaching innovation promoted as part of Horizon 2020 and it was based on previous experiences in the project "A Healthy Diet, your choice" and TANTAKA. A total of 303 students from the Faculty of Pharmacy and Nutrition at the University of Navarra took part in the project, providing dietary advice and training activities at various institutions in Navarre (Spain).
\end{abstract}

\section{Keywords}

Degree in Human Nutrition and Dietetics, service-learning, dietary assessment, training activities. 


\section{Introducción}

El aprendizaje-servicio constituye una metodología docente que combina la docencia con el compromiso social del alumno y permite desarrollar las competencias profesionales a través del servicio voluntario que atienda demandas reales presentes en la comunidad (Gallego, 2014; Malagón, 2009; Martínez, 2008; Rubio, 2011; Santos et al., 2015; Tapia, 2016). Por otro lado, la alimentación es una necesidad básica lo que implica numerosas oportunidades para que los futuros Dietistas-Nutricionistas, aprendan en contextos reales con realidades sociales concretas.

En el marco del Horizonte 2020 de la Universidad de Navarra ${ }^{1}$ (Horizonte 2020, s.f.; Universidad de Navarra, s.f.) dentro de la línea de docencia innovadora, se ha apostado por la metodología aprendizaje-servicio y para impulsar este proyecto se ha creado un grupo promotor de aprendizaje-servicio cuyo principal objetivo es potenciar y apoyar las distintas iniciativas relacionadas con este método en la Universidad de Navarra.

Entre las principales actividades que han surgido de dicho grupo destaca la creación de un sello aprendizajeservicio para fomentar el uso de esta metodología en las asignaturas de la Universidad de Navarra. Este reconocimiento ha quedado plasmado en la guía docente de cada asignatura mediante un distintivo diseñado para

\footnotetext{
${ }^{1}$ www.unav.edu/web/horizonte-2020/proyectos
}

esta ocasión. Un total de 32 asignaturas han conseguido en el curso 2019/2020 este sello, entre ellas tres asignaturas del Grado de Nutrición Humana y Dietética (NHD): Educación Nutricional, Restauración colectiva y Nutrición comunitaria.

El objetivo de esta publicación es describir las experiencias de aprendizaje-servicio desarrolladas en diversas asignaturas del Grado de Nutrición y del Doble Grado FarmaciaNutrición en el periodo 2012-2020 en la Universidad de Navarra, atendiendo a demandas sociales planteadas en su mayoría en el Proyecto "Por una comida sana, tú decides ${ }^{2 "}$ y en el marco de TANTAKA, el Banco de Tiempo Solidario ${ }^{3}$, ambos de la propia universidad

\section{Descripción de las experiencias y resultados}

\subsection{Educación nutricional}

En el curso 2014-2015 se implantó en el Grado de Nutrición el Proyecto de Innovación Docente "Experiencia piloto para la adquisición de habilidades de entrevista y educación nutricional en consulta". Tres fueron los objetivos que pretendía cubrir: integrar las asignaturas de Dietética I (cursada ya en $20 \mathrm{NHD}$ y 40 Doble) y Educación nutricional, realizar una entrevista dietética a personas sanas en un entorno sanitario real y elaborar informe con pautas de mejora del

\footnotetext{
${ }^{2}$ www.unav.edu/web/comida-sana

3 www.unav.edu/web/vidauniversitaria/eventos/solidaridad/que-es-tantaka
} 
patrón alimentario.

Para su puesta en práctica fue necesaria la colaboración de los médicos del Área de Medicina del Trabajo del Servicio de Prevención de Riesgos Laborales de la Universidad. Anualmente, a todos los profesionales se les ofrece realizar un reconocimiento médico considerando los riesgos del puesto de trabajo. Los médicos de empresa del citado centro universitario fueron los encargados de seleccionar a los trabajadores a entrevistar, preferentemente sanos, y supervisar las entrevistas.

Por otro lado, las profesoras que impartían las asignaturas anteriormente mencionadas fueron las encargadas de impartir dos sesiones de formación obligatorias y un seminario de dudas, asesorar en todo momento a los alumnos y revisar y corregir el informe redactado por los alumnos.

Por su parte, los alumnos realizaron por parejas la entrevista dietética personal, elaboraron un informe con las pautas dietéticas a seguir por el trabajador y por último, valoraron de manera anónima el trabajo realizado.

Desde el curso 2014/15, este trabajo obligatorio se ha mantenido en la Guía Docente de la Asignatura, aunque con ligeras modificaciones que no han afectado a lo sustancial del mismo.

En el presente curso académico, el 2019/2020, se ha cambiado el socio comunitario en esta experiencia de aprendizaje-servicio, pasando a ser a partir de ese momento la Fundación
$\mathrm{CORE}^{4}$, una organización sin ánimo de lucro ubicada en Pamplona, cuyo fin es acoger e integrar a los inmigrantes de la ciudad. Para ello, la Universidad de Navarra firmó un convenio con Fundación CORE para que los alumnos citados puedan realizar una entrevista a personas que acuden a la Fundación CORE y voluntariamente quieran colaborar.

Esta entrevista incluye preguntas relativas a los hábitos alimentarios y prácticas dietéticas, así como algunos datos sociodemográficos y datos básicos de salud (peso, estatura, etc.).

En general, en las entrevistas estaba presente el profesor de la asignatura, pero en algunos casos en que no fue posible, se vio necesario grabar las entrevistas en audio o vídeo para que posteriormente las evaluara el profesor.

Los alumnos analizaron los valores recogidos y redactaron un informe proponiendo algunas posibles pautas de mejora. Dicho informe fue entregado al interesado si así lo deseaba, personalmente o por correo en su domicilio. El profesor evaluó el desarrollo de la entrevista y el informe realizado y ambos formaron parte de la nota que el alumno obtuvo en la asignatura.

Este trabajo supuso el $25 \%$ de la nota total, correspondiendo un $12.5 \%$ a las habilidades del alumno en la realización de la entrevista y el $12,5 \%$ a la cumplimentación, análisis e interpretación de la historia dietética y por último a la redacción del informe.

\footnotetext{
${ }^{4}$ www.fundacioncore.wordpress.com
} 
Tanto los alumnos como los profesores estaban sujetos a compromisos de confidencialidad firmados por escrito respecto a los datos personales obtenidos en la entrevista.

Este trabajo siempre ha mantenido una clara coherencia con estos objetivos de la asignatura:

- Adquirir la capacidad de trabajar en equipo y de mejorar sus capacidades de gestión de información, organización, planificación, análisis y síntesis.

- Comunicarse de manera adecuada de modo oral y por escrito.

- Comprender los principios fundamentales de la entrevista.

- Comprender la importancia de la educación nutricional como parte integrante de la Educación para la salud y como pilar insustituible en el tratamiento de distintas enfermedades relacionadas con la alimentación.

Por último, la realización de este trabajo ha permitido trabajar las siguientes competencias y destrezas cívicas:

- Saber comunicarse de una manera constructiva en distintos entornos y mostrar tolerancia.

- Manifestar solidaridad e interés por resolver conflictos.

- Estar dispuesto a superar los prejuicios y respetar las diferencias.

Lo que más valoran los alumnos de esta experiencia de aprendizaje-servicio es que ha sido su primera entrevista en un entorno de trabajo real y una oportunidad de practicar antes de las estancias tuteladas, una experiencia muy positiva y enriquecedora en la que han podido percibir cierta autonomía, el rol del dietista-nutricionista y la clara diferencia entre teoría y práctica.

\subsection{Restauración colectiva}

En este proyecto, han participado los alumnos matriculados en la asignatura Restauración colectiva que se imparte en 3 er curso del Grado en NHD y en $6^{\circ}$ curso del Doble grado en F y NHD. Inicialmente, surgió de manera individual y posteriormente, durante 2 cursos académicos, este proyecto se ha ampliado y realizado conjuntamente con otras asignaturas del Grado, como Seguridad alimentaria y Microbiología de alimentos. Desde el curso 2018-19, con la obtención del sello aprendizajeservicio, este proyecto ha continuado de manera exclusiva en la asignatura.

Desde el comienzo, el objetivo ha sido que los alumnos matriculados en la asignatura preparen e impartan sesiones formativas dinámicas y participativas a profesionales del sector restauración, es decir, a manipuladores de alimentos que trabajan en servicios de alimentación. En concreto, este proyecto se ha realizado en colaboración con el Área de Dietas de la Clínica Universidad de Navarra (CUN) y posteriormente, además con una empresa de restauración colectiva de la zona. 
Esta actividad se inició porque es evidente que existe una necesidad social en relación con la formación en el sector restauración. Teniendo en cuenta la obligatoriedad que marcaba la normativa vigente (Reglamento UE No $852 / 2004$ ), se constató la necesidad de reforzar y actualizar dicha formación en temas de higiene y seguridad alimentaria ya que es frecuente observar que los conocimientos de los manipuladores de alimentos no se traducen en unos adecuados comportamientos y que, además, falta interés y motivación por aprender y mejorar.

Desde el principio, los objetivos que se han perseguido han sido, por un lado, fomentar el interés de los manipuladores de alimentos para mejorar su formación, utilizando diferentes metodologías, y, por otro lado, motivar a los alumnos en su aprendizaje, realizando acciones formativas en entornos profesionales reales.

Para ello, las personas responsables de las han propuesto cada año diferentes temas de interés para sus profesionales, temas que los alumnos preparaban en forma de sesión formativa. Algunos de los temas trabajados han sido los siguientes:

- Beneficios y riesgos de las frituras

- Importancia de un tratamiento térmico correcto

- Mantenimiento de la cadena de frío
- Aspectos clave en relación con la gestión de alérgenos

- Interpretación de resultados microbiológicos.

- Motivación del personal en la aplicación continuada del sistema APPCC.

Este proyecto se lleva realizando desde hace 5 años. Cada curso académico hay una media de 50 alumnos matriculados en Restauración colectiva que se organizan en grupos de 4 a 6 personas. Al comienzo de curso se les informa sobre los diferentes temas que se van a incluir en la formación y cada grupo elige el tema que va a desarrollar. Son los propios alumnos los que se encargaban de buscar y recopilar la información y de diseñar las presentaciones, apoyándose en los contenidos y materiales que se van trabajando en clase. Desde el principio se les indicaba que debían tener muy presente el público al que se dirige su trabajo.

Además, todos los años al comenzar el desarrollo del trabajo, se realizaba una visita al Área de Dietas de la CUN con todos los alumnos, de manera que observaban y comprobaban el trabajo en una cocina hospitalaria. Así mismo, durante este proceso de diseño y desarrollo cada uno de los grupos tiene varias tutorías con las profesoras para supervisar y ajustar, en caso necesario, el contenido y el formato.

Las presentaciones se preparaban en PowerPoint y tenían una duración de unos 15 minutos. Los alumnos aprovechaban para incluir contenidos 
en la sesión formativa a partir de noticias de prensa, o realizar preguntas directas para fomentar la participación. En algunas ocasiones se han llegado a utilizar clickers, dispositivos electrónicos que se entrega a cada uno de los asistentes al comienzo de la sesión de formación de manera que pueden responder en el momento a las preguntas tipo test que se les formulan. Respecto a estas preguntas tipo test los alumnos prepararon 3 opciones de respuesta: una correcta, otra incorrecta y la tercera dudosa de forma que se hiciera pensar a los manipuladores. El curso pasado las sesiones fueron totalmente participativas ya que, tras la sesión formativa, los asistentes realizaron un Kahoot preparado por los alumnos.

Esta actividad se incluye en la evaluación de la asignatura, suponiendo el $25 \%$ de la nota final. En este porcentaje se valoraba tanto la búsqueda de información, preparación y diseño del tema elegido (entre los sugeridos por las profesoras) como su exposición a los manipuladores de alimentos reales a lo largo del semestre.

Hasta la fecha, los principales resultados obtenidos ponen de manifiesto que los manipuladores de alimentos valoran positivamente estas sesiones de formación y que se consigue una implicación y participación en las actividades propuestas por los alumnos. De hecho, en la evaluación realizada este último año, tanto la expresión oral de los alumnos como la adaptación de los contenidos fueron valorados con un 8,1 sobre 10 . Los alumnos por su parte indicaron que les supone un reto adaptar el lenguaje científico al que están acostumbrados en las aulas, a un lenguaje más divulgativo. Además, consideran una gran oportunidad poder poner en práctica su propio trabajo en un entorno profesional real y valoran positivamente tanto el contenido de las presentaciones como el formato en que las realizan.

Esta actividad está en coherencia con los objetivos de la asignatura ya que una de las tareas del dietista-

nutricionista que trabaja en el sector de la restauración colectiva es la formación de manipuladores de alimentos. Con este proyecto, los alumnos se ponen en el papel del profesional a la vez que va asimilando algunos de los contenidos de la asignatura.

En relación con las competencias sociales y destrezas cívicas que adquiere el alumno mediante esta actividad de aprendizaje-servicio, destacan la empatía con el colectivo que recibe la formación y la responsabilidad en la mejora del desempeño profesional de dicho colectivo. Con respecto a las competencias profesionales que el alumno desarrolla se incluye el trabajo en equipo, la capacidad de búsqueda de información, la capacidad de comunicar en un lenguaje divulgativo y no excesivamente científico, así como el compromiso por el trabajo bien hecho y de calidad.

Además, durante el proceso de preparación de la sesión formativa el alumno tiene ocasión de reflexionar y repensar la actividad tanto en el propio grupo de trabajo, donde ponen en 
común aspectos de contenido y de diseño pensando en el grupo diana de la formación, como en las tutorías que se organizan con las profesoras de la asignatura.

\subsection{Nutrición comunitaria}

La asignatura de Nutrición Comunitaria es una asignatura obligatoria que aborda cómo mejorar el estado nutricional y de salud a nivel individual y en la comunidad, lo que implica un marco idóneo para la puesta en marcha de actividades de aprendizaje-servicio en el ámbito de la Nutrición coherentes con los objetivos de la asignatura.

De manera específica, se trabajan las competencias del Grado relacionadas con:

- El análisis, planificación, y evaluación de programas de intervención en alimentación.

- Diseño y puesta en marcha programas de educación dietético-nutricional intervención en proyectos de promoción, prevención y protección con un enfoque comunitario.

Desde el Curso 2018-2019, esta asignatura incluye la realización de una actividad obligatoria y evaluable de aprendizaje-servicio, que permite trabajar estas competencias en entornos reales y en población diana de riesgo nutricional. En ella, los alumnos en grupos reducidos deben diseñar y aplicar de forma tutelada un proyecto de intervención en nutrición comunitaria adaptada a las necesidades formativas de diversas instituciones que atienden a colectivos vulnerables o en situación de riesgo de exclusión social.

Hasta la fecha se han realizado proyectos de aprendizaje-servicio en las siguientes entidades: Comité Español de Ayuda al Refugiado en Navarra, Banco de Alimentos de Navarra, Fundación CORE, Red Madre, Asociación Bizilaguna y Fundación Profesionales Solidarios. Las áreas formativas más demandadas fueron:

- Compra saludable y económica

- Etiquetado nutricional

- Desperdicio alimentario

- Grupos de alimentos

- Dieta Mediterránea asequible

- Alimentación en distintos grupos de población: embarazadas y madres lactantes, niños y personas mayores.

En base a estos temas se realizaron talleres de 90 minutos en las propias entidades y en caso de limitación de aforo, se podían a disposición aulas en la Universidad. En el Banco de alimentos, además de acciones formativas se realizó asesoramiento técnico para la gestión de stocks y preparación más eficiente desde el punto de vista nutricional, de los lotes que distribuyen a las organizaciones asociadas al reparto de alimentos en Navarra.

Todos los grupos debían entregar la propuesta de contenidos en una fecha cerrada y se les daba feedback con 
correcciones y sugerencias, antes de la puesta en marcha

Para la adecuada supervisión de los proyectos, se organizaron 5 seminarios de 2 horas de duración donde los alumnos pueden trabajar en sus proyectos y consultar dudas. En algunas instituciones como Fundación CORE o Banco de alimentos, se realizaron varios talleres, por lo que los grupos implicados debían coordinar los contenidos de sus respectivos talleres.

Además de las competencias propias de esta asignatura, mediante esta actividad se fomentó la comunicación directa entre los alumnos y los técnicos responsables en las entidades para que con el objetivo de que el alumno pudiera adquirir también habilidades comunicativas y de gestión con instituciones.

Esta actividad de aprendizaje-servicio supone un $20 \%$ de la calificación final de la asignatura y tenía en cuenta los siguientes criterios de evaluación:

- Que los contenidos fueran técnicamente apropiados, originales y adaptados al nivel de la población diana

- En las sesiones se evaluaba la claridad, los recursos utilizados y la capacidad para ajustarse al tiempo establecido y para resolver dudas. En el caso de que hubiera preguntas complicadas los profesores tutores tenían indicación de intervenir.

- Y por último, también se tenía en cuenta la valoración por parte de los responsables de las entidades.

Además, de la evaluación propia de la asignatura, se incluyó la realización de un ANÁLISIS DAFO, para fomentar la reflexión en los alumnos sobre esta actividad. Cabe resaltar que las principales debilidades que indicaron los alumnos fueron, por un lado, el escaso o ningún contacto previo población en riesgo de exclusión social, y por otro, la dificultad para adaptar el contenido y el lenguaje a una población diana multicultural.

La implantación de esta metodología de aprendizaje-servicio, ha facilitado que el alumno adquiera ciertas competencias sociales y destrezas cívicas. Entre ellas, destacan: la sensibilización y concienciación de las necesidades formativas de colectivos vulnerables o en riesgo de exclusión social. Además, saber comunicarse de una manera constructiva en distintos entornos y mostrar tolerancia, manifestar solidaridad e interés por resolver conflictos. Y, por último, estar dispuesto a superar los prejuicios y respetar las diferencias.

Por último, hay que mencionar que desde el año 2012, los alumnos del Grado de Nutrición y del Doble Grado Farmacia-Nutrición han participado en experiencias voluntarias previas, sin ser propiamente experiencias de aprendizaje-servicio, gracias a la colaboración de 6 profesores. A continuación se describen las 13 actividades realizadas, agrupadas en 5 áreas de actuación, con un total de 7 partners. 
- Asesoramiento dietético y promoción de elecciones saludables en las cafeterías y máquinas expendedoras del campus anualmente con motivo del Día de la Alimentación saludable y Día del Deporte.

- Trabajo de Fin de Grado: para conocer los hábitos alimentarios de los usuarios de máquinas vending y cafeterías del campus.

- Visita al Banco de Alimentos de Navarra, preparación de diversos materiales escritos y selección de recursos para su web, asesoramiento sobre los productos aconsejados en las Recogidas y en gestionar los stocks y reparto de lotes según las frecuencias de consumo de alimentos recomendadas en adultos y niños.

- Preparar material de apoyo para clases de cocina sobre cuestiones básicas de los distintos grupos de alimentos en Fundación CORE.

- Impartir sesiones teóricoprácticas sobre aspectos básicos de Alimentación y Dieta Mediterránea dirigidas a inmigrantes atendidos en Fundación CORE, a población en riesgo de inclusión social de Villa Teresita ${ }^{5}$ y al Comité Español de Ayuda al Refugiado con sede en Navarra6.

\footnotetext{
${ }^{5}$ www.villateresita.org/

${ }^{6}$ www.cear.es
}

\section{Conclusión}

Desde el año 2012 y hasta el curso $2019 / 2020$ en total se han realizado un total de 121 entrevistas de asesoramiento dietético personalizado y 77 sesiones formativas.

Los alumnos implicados valoran las actividades de aprendizaje-servicio mencionadas como una experiencia positiva, innovadora, enriquecedora y que les ha permitido alcanzar nuevas competencias más allá del curriculum académico. Por parte de los profesores implicados, se constatan las ventajas de acercar al alumno a entornos reales que faciliten adquisición de competencias del dietista-nutricionista y que fomenten su formación en responsabilidad social. Así, en sus propias palabras "las experiencias de aprendizaje-servicio forman y transforman no sólo a los alumnos, sino también a los profesores", "el aprendizaje-servicio fomenta la responsabilidad social en los alumnos y permite trabajar competencias profesionales del Dietista-Nutricionista en un entorno real" y "el aprendizajeservicio es una experiencia estupenda para formar universitarios integrales y competentes capaces de transformar el mundo".

En conclusión, las experiencias de aprendizaje-servicio descritas en esta publicación, han permitido aplicar lo aprendido en torno a la alimentación y nutrición en las aulas a situaciones reales, y han contribuido a que los alumnos del Grado de NHD y del Doble Grado en Farmacia NHD, reflexionen sobre las necesidades de diferentes colectivos, y por último, han promovido

Santiago, S.; Garayoa, R. y Zazpe, I. (2021). Aplicación de la metodología aprendizaje-servicio en el Grado de Nutrición Humana y Dietética de la Universidad de Navarra. RIDAS, Revista Iberoamericana de Aprendizaje Servicio, 11, 128-138. DOI10.1344/RIDAS2021.11.9 
el compromiso social del alumno y el desarrollo de competencias profesionales y sociales

\section{Referencias bibliográficas}

Gallego, M. (2014). El aprendizajeservicio como estrategia metodológica en la universidad. Revista Complutense de Educación, 25(1), 95-113. doi:

10.5209/rev_RCED.2014.v25.n1.41157

Horizonte 2020. (s.f.). Plan de Incentivación para la participación española en Horizonte Europa. Ministerio de Innovación, Ciencia y Universidades.

https://eshorizonte2020.es/

Malagón, L. (2009). La relación universidad-sociedad: una visión crítica. Revista Perspectivas Educativas, 2, 1750. Recuperado de http://revistas.ut.edu.co/index.php/per spectivasedu/article/view/787/615

Martínez, M. (2008). Aprendizaje servicio y responsabilidad social de las universidades. Barcelona, España:

Octaedro.

Rubio, L. (2011). Aprendizaje- servicio: aterrizaje entre teoría y práctica. Aula de Innovación Educativa, (203-204), 34-37. Recuperado de https://mestreacasa.gva.es/c/documen $\mathrm{t}$ library/get file?folderId $=5000052935$ $\underline{04 \& \text { name }=\text { DLFE-274011.pdf }}$

Santos, M., Sotelino, A. y Lorenzo, M. (2015). Aprendizaje-servicio y misión cívica de la universidad: una propuesta de desarrollo. Barcelona, España:

Octaedro.
Tapia, M. N. (2016). Inserción curricular del aprendizaje- servicio en la Educación Superior. Ciudad Autónoma de Buenos Aires, Argentina:CLAYSS.

Universidad de Navarra. (s.f.). Horizonte 2020. Metodologías docentes. https://www.unav.edu/web/horizonte2020/metodologias-docentes Aprendizaje Servicio, 11, 128-138. DOI10.1344/RIDAS2021.11.9 